\title{
A Finite-Difference Model to Study the Elastic-Wave Interactions with Buried Land Mines
}

\author{
Christoph T. Schröder and Waymond R. Scott, Jr.
}

\begin{abstract}
A two-dimensional (2-D) finite-difference model for elastic waves in the ground has been developed. The model uses the equation of motion and the stress-strain relation, from which a first-order stress-velocity formulation is obtained. The resulting system of equations is discretized using centered finite-differences. A perfectly matched layer surrounds the discretized solution space and absorbs the outward traveling waves. The numerical model is validated by comparison to an analytical solution. The numerical model is used to study the interaction of elastic waves with a buried land mine. It is seen that the presence of an air-chamber within the mine gives rise to resonant oscillations that are clearly visible on the surface above the mine. The resonance is shown to be due to flexural waves being trapped within the thin layer between the surface of the ground and the air chamber of the mine. The numerical results are in good qualitative agreement with experimental observations.
\end{abstract}

Index Terms-Acoustic, elastic wave, FDTD, finite-difference, land mine.

\section{INTRODUCTION}

$\mathbf{I}$ $\mathrm{N}$ A NEW technique, elastic and electromagnetic waves are used in a synergistic manner to detect buried land mines [1], [2]. Within this technique, elastic waves interact with a mine and cause the ground above the mine to vibrate. A radar detects these vibrations and, thus, the mine. To explore the mine-wave interactions, a numerical finite-difference model for elastic waves in the ground has been developed.

The equation of motion and the stress-strain relation, together with a constitutive relation, form a set of first-order partial differential equations that completely describe the elastic wave motion in a medium. Introducing finite differences, this set of equations can be discretized and adapted to the finite-difference time-domain modeling scheme, obtaining a second-order accurate stress-velocity formulation. Assuming that the field is known at one initial time $t_{0}$, this numerical scheme provides the field values at any later time $t>t_{0}$. The finite-difference model has been implemented in two dimensions. The solution space is discretized and a staggered finite-difference grid is introduced. The grid is surrounded by a perfectly matched layer, that absorbs the outward traveling waves. The numerical model has been validated by comparison to an analytical solution. The analytical solution for elastic waves in a homogeneous semi-infinite half-space is obtained in the form of an integral equation.

Manuscript received September 4, 1999; revised February 16, 2000. This work was supported in part by the OSD MURI program, U.S. Army Research Office, Washington, DC, Contract DAAH04-96-1-0448.

The authors are with the School of Electrical and Computer Engineering, Georgia Institute of Technology, Atlanta, GA 30332 USA (e-mail christoph.schroeder@ee.gatech.edu).

Publisher Item Identifier S 0196-2892(00)05890-3.

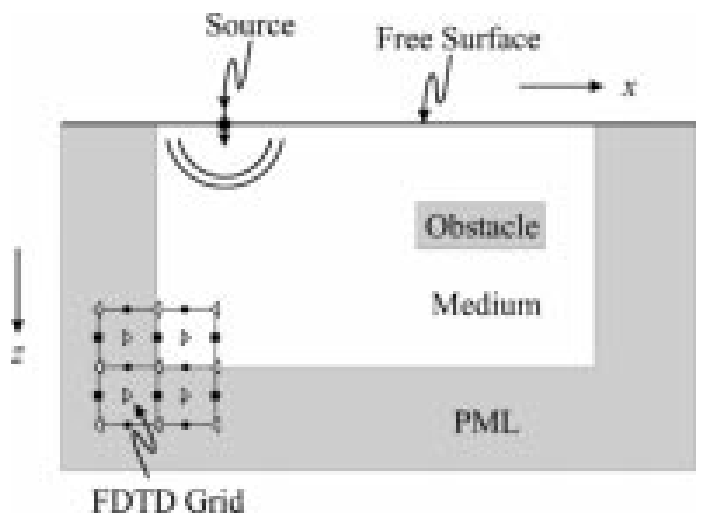

Fig. 1. Finite-difference model.

Excellent agreement is seen between the numerical model and the analytical solution.

By using the numerical model, the interaction of elastic waves with a buried antipersonnel mine is explored. Results for two simple models of a TS-50 antipersonnel mine are presented in this work: one containing an air-filled chamber and one without an air-filled chamber. The air-filled chamber is introduced to approximate the complex structure of a real mine, which contains explosives as well as chambers bearing the triggering mechanism etc. The results obtained with the numerical model are in remarkably good agreement with experimental observations even though the model is 2-D, whereas the experimental set-up is 3-D. In both experiments and numerical simulations, a strong resonance is observed at the mine. This resonance is shown to be due to flexural waves being trapped within the thin layer between the surface of the ground and the air chamber of the mine. In the experiments, the Rayleigh surface waves are seen to disperse while traveling along the surface. Assuming a depth-dependent shear wave speed, this effect can be also modeled numerically.

\section{Two-Dimensional (2-D) NUMERICAL MODEL}

\section{A. Finite-Difference Model}

Fig. 1 shows the 2-D finite-difference model. The ground is modeled as an isotropic, lossless semi-infinite half-space. A normal point source is located on the free surface, exciting longitudinal (pressure) and transverse (shear) waves in the $x-z$-plane. The wave fields are invariant in the $y$ direction. At $z=0$, a free surface bounds the solution space. A perfectly matched layer (PML) terminates the solution space at the remaining edges, absorbing all outward traveling waves. The space is discretized using a staggered finite-difference grid. 
A first-order velocity-stress formulation is used for the finitedifference model [3]-[5]. Here, the equation of motion and the stress-strain relation are discretized, leading to a system of firstorder partial differential equations. Since only field components in the $x$ - $z$-plane are excited, the only nonzero field components are the three unknown stress components $\tau_{x x}, \tau_{x z}, \tau_{z z}$, and the two unknown particle velocities $v_{x}$ and $v_{z}$. Due to the invariance in the $y$-direction, all derivatives with respect to $y$ vanish. The wave motion is then completely described by a system of the following five partial differential equations:

$$
\begin{aligned}
\rho \frac{\partial v_{x}}{\partial t} & =\frac{\partial \tau_{x x}}{\partial x}+\frac{\partial \tau_{x z}}{\partial z} \\
\rho \frac{\partial v_{z}}{\partial t} & =\frac{\partial \tau_{x z}}{\partial x}+\frac{\partial \tau_{z z}}{\partial z} \\
\frac{\partial \tau_{x x}}{\partial t} & =(\lambda+2 \mu) \frac{\partial v_{x}}{\partial x}+\lambda \frac{\partial v_{z}}{\partial z} \\
\frac{\partial \tau_{z z}}{\partial t} & =(\lambda+2 \mu) \frac{\partial v_{z}}{\partial z}+\lambda \frac{\partial v_{x}}{\partial x} \\
\frac{\partial \tau_{x z}}{\partial t} & =\mu\left(\frac{\partial v_{x}}{\partial z}+\frac{\partial v_{z}}{\partial x}\right)
\end{aligned}
$$

where $\rho$ is the material density, and $\lambda$ and $\mu$ are Lame's constants. These equations are discretized using centered finite differences. By introducing the finite differences in space $\Delta x$ and $\Delta z$ and in time $\Delta t,(1)$ and (3), for example, can be discretized as

$$
\begin{aligned}
\rho \frac{\left.V_{x}^{k+0.5}\right|_{i, j-0.5}-\left.V_{x}^{k-0.5}\right|_{i, j-0.5}}{\Delta t} \\
=\frac{\left.T_{x x}^{k}\right|_{i+0.5, j-0.5}-\left.T_{x x}^{k}\right|_{i-0.5, j-0.5}}{\Delta x} \\
\quad+\frac{\left.T_{x z}^{k}\right|_{i, j}-\left.T_{x z}^{k}\right|_{i, j-1}}{\Delta z}
\end{aligned}
$$

and

$$
\begin{aligned}
& \frac{\left.T_{x x}^{k+1}\right|_{i+0.5, j-0.5}-\left.T_{x x}^{k}\right|_{i+0.5, j-0.5}}{\Delta t} \\
& =(\lambda+2 \mu) \frac{\left.V_{x}^{k+0.5}\right|_{i+1, j-0.5}-\left.V_{x}^{k+0.5}\right|_{i, j-0.5}}{\Delta x} \\
& \quad+\lambda \frac{\left.V_{z}^{k+0.5}\right|_{i+0.5, j}-\left.V_{z}^{k+0.5}\right|_{i+0.5, j-1}}{\Delta z} .
\end{aligned}
$$

Here, the capital letters mark the numerical value of the correspondent field component at a discrete location in space and time. For example, $\left.V_{x}^{k+0.5}\right|_{i, j-0.5}$ stands for the numerical value of the particle velocity $v_{x}$ at the position $(x, z)=(i \Delta x,(j-0.5) \Delta z)$ at the time $t=(k+0.5) \Delta t$. Knowing $V_{x}^{k-0.5}, T_{x x}^{k}$, and $T_{x z}^{k}$, (6) can be solved for $V_{x}^{k+0.5}$, i.e. at the incremental time $t=(k+0.5) \Delta t$

$$
\begin{aligned}
\left.V_{x}^{k+0 . \tilde{5}}\right|_{i, j-0.5}= & \left.V_{x}^{k-0.5}\right|_{i, j-0.5} \\
& +\frac{\Delta t}{\rho \Delta x}\left(\left.T_{x x}^{k}\right|_{i+0.5, j-0.5}-\left.T_{x x}^{k}\right|_{i-0 . \tilde{5}, j-0.5}\right) \\
& +\frac{\Delta t}{\rho \Delta z}\left(\left.T_{x z}^{k}\right|_{i, j}-\left.T_{x z}^{k}\right|_{i, j-1}\right) .
\end{aligned}
$$

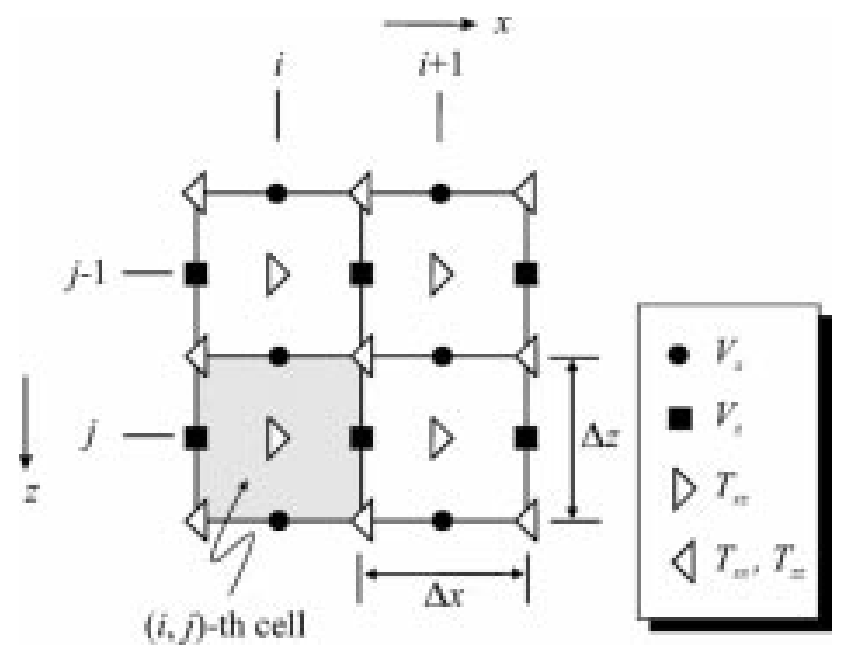

Fig. 2. Portion of the finite-difference grid. The field components are not known at the same locations but are offset by $\Delta x / 2$ and $\Delta z / 2$.

Similarly, $T_{x x}^{k+1}$ is obtained from (7)

$$
\begin{aligned}
\left.T_{x x}^{k+1}\right|_{i+0 . \tilde{s}, j-0 . \tilde{s}} & \left.T_{x x}^{k}\right|_{i+0.5, j-0.5}+(\lambda+2 \mu) \frac{\Delta t}{\Delta x} \\
& \cdot\left(\left.V_{x}^{k+0.5}\right|_{i+1, j-0.5}-\left.V_{x}^{k+0.5}\right|_{i, j-0.5}\right) \\
& +\lambda \frac{\Delta t}{\Delta z}\left(\left.V_{z}^{k+0.5}\right|_{i+0.5, j}-\left.V_{z}^{k+0.5}\right|_{i+0.5, j-1}\right) .
\end{aligned}
$$

In the same manner, discretized equations can be obtained for all field components.

The discretization leads to the characteristic staggered finitedifference grid. Fig. 2 depicts the position of the field components in the finite-difference grid. In this grid, the velocity components and the stress components are not known at the same position in time and space but are offset by $\Delta t / 2$ in time and by $\Delta x / 2$ and $\Delta z / 2$ in space. The offset in time leads to the so-called leapfrog scheme. In the leapfrog scheme, the field components are updated sequentially in time. The velocity components are calculated first, then the stress components from the velocity components, the velocity components again using the stress components, and so on. Thus, knowing the field components throughout the entire space at the time $t_{0}-0.5 \Delta t$ and $t_{0}$, respectively, the field components can be determined for all later times $t>t_{0}$.

When implementing the finite-difference scheme, boundary conditions must be treated in a special manner. Three different kinds of boundaries arise: the source point, the internal boundaries (i.e., boundaries within the medium marked by a change in material properties), and the external boundaries (i.e., the grid edges).

A normal point source is implemented on the free surface. The normal stress component $\tau_{z z}$ is excited by a differentiated Gaussian pulse. An additive source is used. For each time step, the value of the excitation is added to the value calculated with the finite-difference scheme. 
The conditions at internal boundaries (i.e., at the interfaces between different media) are usually satisfied implicitly. However, to ensure numerical stability, the material properties must be averaged for components on the boundary. While the material density $\rho$, appearing in the equation of motion, is averaged directly, the inverse of Lame's constants. For transitions between similar materials, the averaging may be omitted. However, it is necessary at an interface between media with greatly different material properties (for example, at an air-solid interface) in order to maintain stability [3].

Four external boundaries arise at the four outer grid edges. At its upper edge, the half-space is bounded by a free surface. Due to the continuity of normal stress, the normal stress components vanish at a free surface. In order to satisfy this condition, an extra row must be inserted into the finite-difference grid one step beyond the free-surface boundary. By using this extra row, all stress components are forced to zero on the free surface [3].

In order to model the semi-infinite half-space, all waves that are reaching the three remaining outer grid edges must be perfectly transmitted and absorbed. The boundary condition that does this most accurately is the PML boundary condition, first introduced by Berenger [6] and adapted to elastodynamics by Chew and Liu [7]. In continuous space, a nonphysical splitting of the wave fields allows the introduction of a lossy boundary layer that is perfectly matched to the solution space. It has been shown that an arbitrarily polarized wave incident on this PML is perfectly transmitted. The wave experiences the exact same phase velocity and wave impedance as in the solution space, while rapidly decaying along the axis normal to the PML/medium interface. However, in discrete space, the lossy layer will not be perfectly matched to the solution space, and slight reflections occur at the interface. To keep these reflections small, a tapered loss profile is chosen within the PML

$$
\sigma(i)=\sigma_{\max } \cdot\left(\frac{i-i_{\mathrm{PML}}}{N_{\mathrm{PML}}}\right)^{m}
$$

where $m \approx 2.1$ and $\sigma_{\max }=0.1 N_{\text {PML }} / \Delta t ; N_{\text {PML }}$ is the thickness of the PML in basis cells, and $i-i_{\mathrm{PML}}$ indicates the position within the PML. This loss profile (with a slightly different $\sigma_{\max }$ ) has been found to yield good performance in electromagnetic finite-difference modeling [8]. For this work, a thickness of ten cells has been found sufficient, yielding an attenuation of the incident waves of more than $70 \mathrm{~dB}$.

\section{B. Validation of Finite-Difference Results}

An analytical solution for the particle displacement fields in a semi-infinite homogeneous half-space subjected to a normal harmonic line-load of finite width can be derived [9], [10]. Using a Fourier transform method, the particle displacement is obtained as an integral equation and can be computed using numerical integration [3].

The integral to be solved contains two poles and four branch cuts. Furthermore, the integration range is infinite and extends from $-\infty$ to $\infty$. While the branch cuts do not impose a problem during numerical integration, the poles cannot be integrated numerically. To account correctly for the pole contributions, the

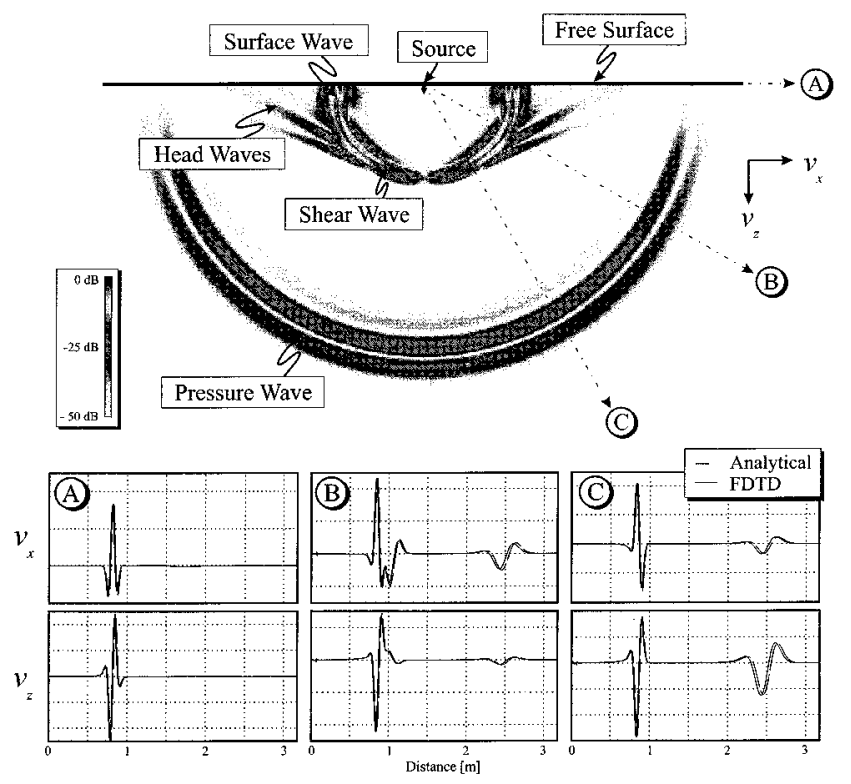

Fig. 3. Plot of the magnitude of the elastic waves in a half-space subjected to a normal line-load at $t=10 \mathrm{~ms}$.

poles must be subtracted from the integral and integrated analytically. To accelerate the integration, the infinite integration range may be truncated. However, for this to be possible, the integrand has to converge sufficiently fast to zero. In order to accelerate the convergence of the integral, an asymptotic approximation for the tails of the integrals is derived. This asymptotic approximation is subtracted from the integrals and integrated analytically.

To obtain the particle displacement in the time-domain, the displacement field must be Fourier-transformed. Since the analytical solution is to be compared to the finite-difference result, where the particle velocity is determined, the particle displacement is differentiated with respect to time, and the particle velocity is obtained.

The integral is computed on a $600 \times 600$ grid with a grid spacing of $0.53 \mathrm{~cm}$. Noting that the wave fields are symmetric to the vertical axis passing through the source, this yields an effective solution space of $6.37 \mathrm{~m} \times 3.18 \mathrm{~m}$. The finite-difference simulation is performed using a grid with $1239 \times 620$ nodes, incorporating a PML layer with a thickness of 20 cells. The finite-difference grid has the same dimensions and the same spacing as the grid used for the analytical result but does not exploit the symmetry and is consequently twice as big. The time step is chosen to be $\Delta t=1.06 \cdot 10^{-5} \mathrm{~s}$, and 1200 time steps are needed to calculate the wave fields up to $t=10 \mathrm{~ms}$. The computations for the analytical solution took about 24 times longer than those for the numerical simulation.

In Fig. 3, the elastic waves due to a normal line-load on a free surface are shown. A differentiated Gaussian pulse with a center frequency of $400 \mathrm{~Hz}$ is launched at $t=0 \mathrm{~s}^{1}$ from a source located on the free surface. In the upper half of Fig. 3, the magnitude of the particle velocity field at $t=10 \mathrm{~ms}$ is shown. A pressure, a shear, and a surface wave are seen to propagate. The pressure wave is the fastest of the three. The surface wave

${ }^{1}$ The center of the pulse is located at $t=0 \mathrm{~s}$. 


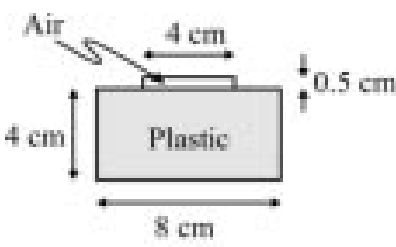

(a)

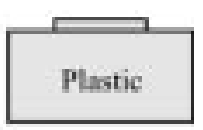

(b)
Fig. 4. Simple model of the TS-50 antipersonnel mine (a) with and (b) without an air-filled chamber.

is propagating slightly slower than the shear wave and contains the most energy. A plane shear wave arises at the free surface, induced by the pressure wave. This wave is called a head wave. Head waves are downwardly directed shear waves generated by the passage of bulk waves along the free surface. Two more plane waves can be seen, which propagate at a steeper angle than the head wave induced by the pressure wave.

In Fig. 3, the particle velocity components $v_{x}$ and $v_{z}$, according to the numerical and analytical solution, are plotted along the three section lines through the ground, denoted by A, $\mathrm{B}$, and $\mathrm{C}$. The agreement of numerical and analytical solutions is excellent. It can be seen that the finite-difference model predicts waves that travel slightly slower than those of the analytical solution. This is due to the well-known fact that the numerical phase velocity in the discrete finite-difference grid will come out to be slightly smaller than in continuous space [11].

The head waves obtained both with the finite-difference model and in the analytical solution have also been observed experimentally by the authors and others. In [12], a seismic field survey in a region with extremely high Poisson's ratio is described. In these measurements, head waves are detected that are very similar to the head waves observed here.

\section{INTERACTION OF ELASTIC WAVES WITH A TS-50 ANTIPERSONNEL MINE}

The interaction of elastic waves with a TS-50 antipersonnel mine, buried in sand, has been investigated. Two simple models for the TS-50 mine are used. In the first model [Fig. 4(a)], a small chamber filled with air is located on top of the mine's main chamber containing plastic explosives. The second model does not contain an air-filled chamber [Fig. 4(b)]. By including an air-filled chamber into the mine model, the effects of a real mine are approximated. A real mine is a complex mechanical structure with a flexible case, a trigger mechanism, air chambers, etc. Fig. 5 depicts a simplified cross sectional drawing of a real TS-50 antipersonnel mine. The elastic properties of the materials used for the numerical simulation are summarized in Table I. The parameters for the sand and for the plastic were measured experimentally by the authors, whereas the properties of the air are obtained from the literature.

A differentiated Gaussian pulse with a center frequency of $450 \mathrm{~Hz}$ is launched at $t=0 \mathrm{~s}$ from a source on the free surface. The left edge of the mine is located at a distance of $85 \mathrm{~cm}$ from the source. Its upper edge lies $1.5 \mathrm{~cm}$ beneath the surface of the ground. The space step within the finite-difference grid is set to $\Delta x=\Delta z=0.5 \mathrm{~cm}$. The time step is chosen to be $\Delta t=\Delta x /\left(\sqrt{2} c_{p, \max }\right)=1.309 \cdot 10^{-6} \mathrm{~s}$ and thus fulfills the

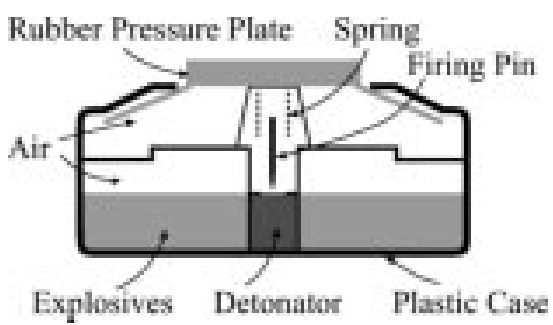

Fig. 5. Cross-sectional drawing of a TS-50 antipersonnel mine.

TABLE I

PARAMETERS USED FOR FINITE-DIFFERENCE SIMULATION

\begin{tabular}{llll}
\hline Sand & Shear wave velocity & $c_{s, \text { sand }}$ & $87 \mathrm{~m} / \mathrm{s}$ \\
& Pressure wave velocity & $c_{p \text {,sand }}$ & $250 \mathrm{~m} / \mathrm{s}$ \\
& Material density & $\rho_{\text {sand }}$ & $1400 \mathrm{~kg} / \mathrm{m}^{3}$ \\
\hline \multirow{2}{*}{ Mine } & Shear wave velocity & $c_{s, \text { mine }}$ & $1100 \mathrm{~m} / \mathrm{s}$ \\
& Pressure wave velocity & $c_{p, \text { mine }}$ & $2700 \mathrm{~m} / \mathrm{s}$ \\
& Material density & $\rho_{\text {mine }}$ & $1200 \mathrm{~kg} / \mathrm{m}^{3}$ \\
\hline \multirow{2}{*}{ Air } & Shear wave velocity & $c_{s, \text { air }}$ & $0 \mathrm{~m} / \mathrm{s}$ \\
& Pressure wave velocity & $c_{p, \text { air }}$ & $330 \mathrm{~m} / \mathrm{s}$ \\
& Material density & $\rho_{\text {air }}$ & $1.3 \mathrm{~kg} / \mathrm{m}^{3}$ \\
\hline
\end{tabular}

Courant condition. The elastic wave fields are computed on a grid containing $350 \times 200$ cells, including a PML with a thickness of ten cells and, consequently, yielding an effective solution space of $165 \mathrm{~cm} \times 95 \mathrm{~cm}$. The computation time was approximately $90 \mathrm{~min}$ for 22000 time steps on a $450 \mathrm{MHz}$ PC.

Fig. 6 shows the elastic wave fields on a cross section through the ground for (a) the model with the air-filled chamber and (b) the model without the air-filled chamber. The magnitude of the particle velocity is plotted on a logarithmic scale. The particle velocity field is shown at four different times: $t=4 \mathrm{~ms}, t=10$ $\mathrm{ms}, t=11 \mathrm{~ms}$, and $t=16 \mathrm{~ms}$.

At $t=4 \mathrm{~ms}$, a pressure wave $(\mathrm{P})$, a shear wave (S), a Rayleigh surface wave $(\mathrm{R})$, and head waves $(\mathrm{H})$ are seen to propagate. The pressure wave, the fastest of the waves, just hits the mine. For the mine with the air-filled chamber, some energy is seen to be trapped between the surface and the mine, while for the mine without the air-filled chamber, no strong interaction occurs. At $t=10 \mathrm{~ms}$ and $t=11 \mathrm{~ms}$, the surface wave (S) hits the mine and is partially transmitted (R) and partially reflected (rR). At $t=16 \mathrm{~ms}$, the waves reflected from the mine are clearly seen [these are reflected Rayleigh waves (rR), a reflected shear wave $(\mathrm{rS})$, and a reflected pressure wave (rP)]. For the mine without the air-filled chamber, no energy is seen to remain at the mine. However, for the mine with the air-filled chamber, energy is seen to be trapped above the mine and to be radiating waves, even though the incident wave has passed by.

In Fig. 7, waterfall graphs of the vertical particle velocity $v_{z}$ at the surface are shown for (a) the mine with the air-filled chamber and (b) the mine without the air-filled chamber. Here, $v_{z}$ is plotted as a function of time and vertically offset by the distance from the source. The slope of the traveling waves in the graph indicates the wave speed. Thus, by looking at their slope, the different waves can be distinguished. A Rayleigh surface wave $(\mathrm{R})$ and a pressure wave $(\mathrm{P})$ are seen to be incident 


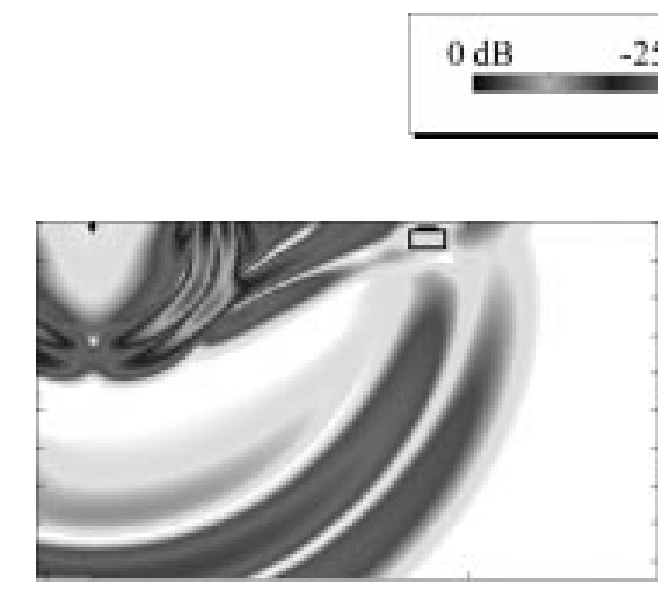

$$
t=4 \mathrm{~ms}
$$
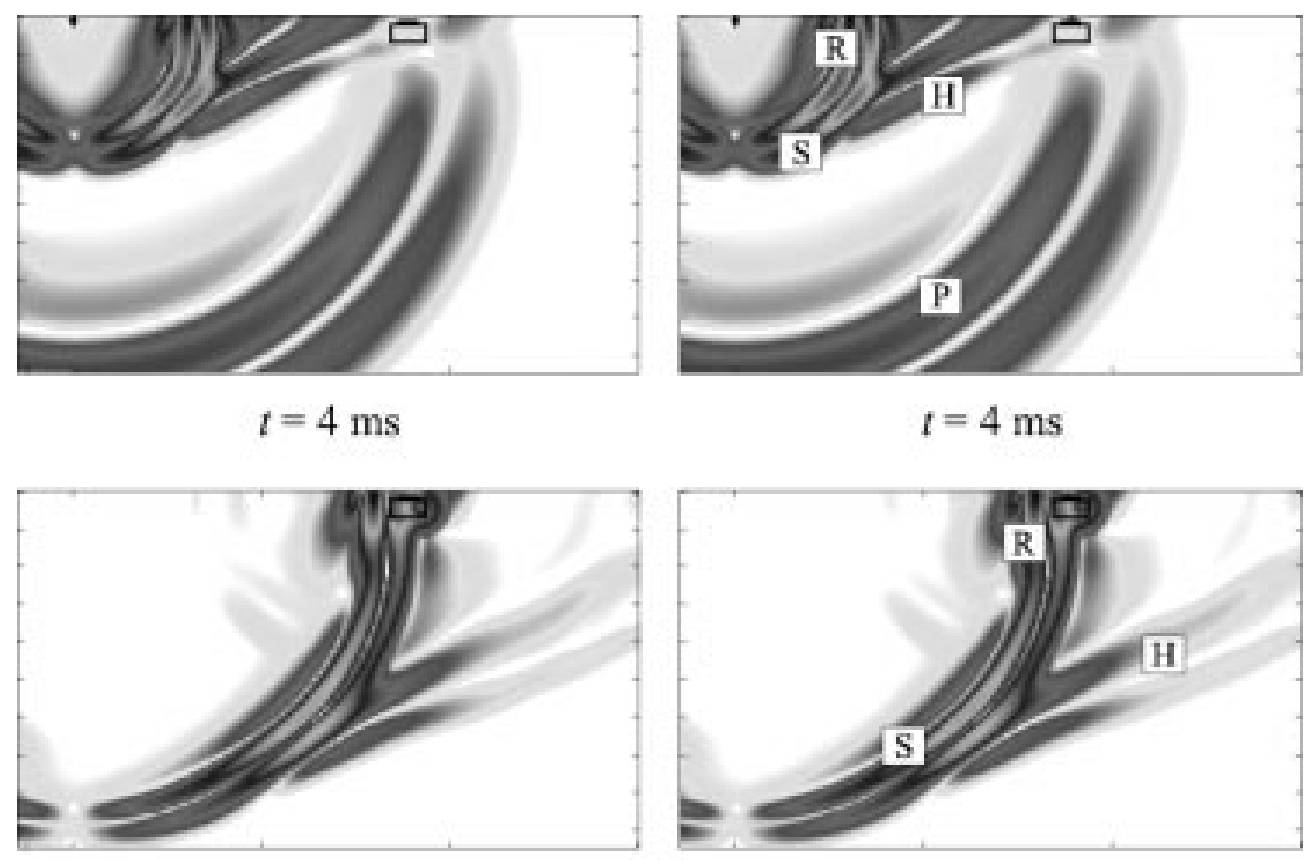

$$
t=10 \mathrm{~ms}
$$

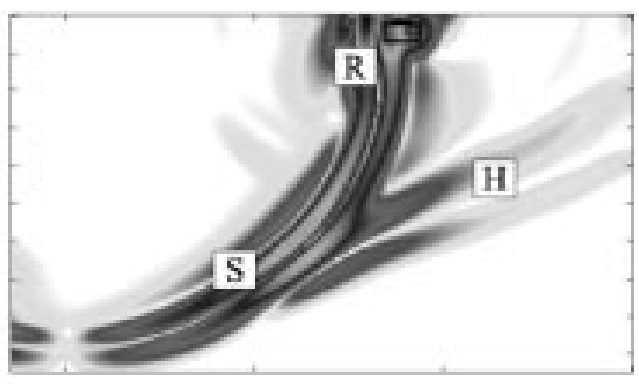

$$
t=10 \mathrm{~ms}
$$

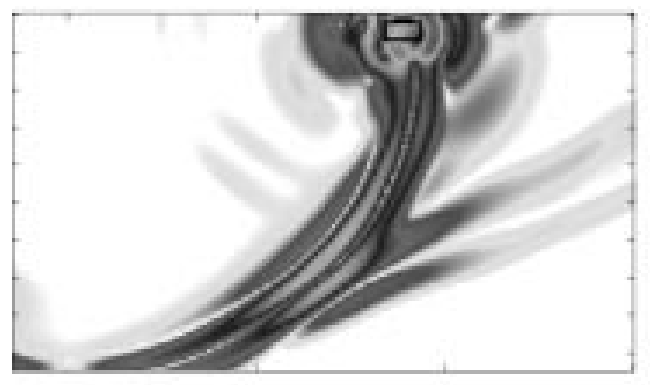

$$
t=11 \mathrm{~ms}
$$
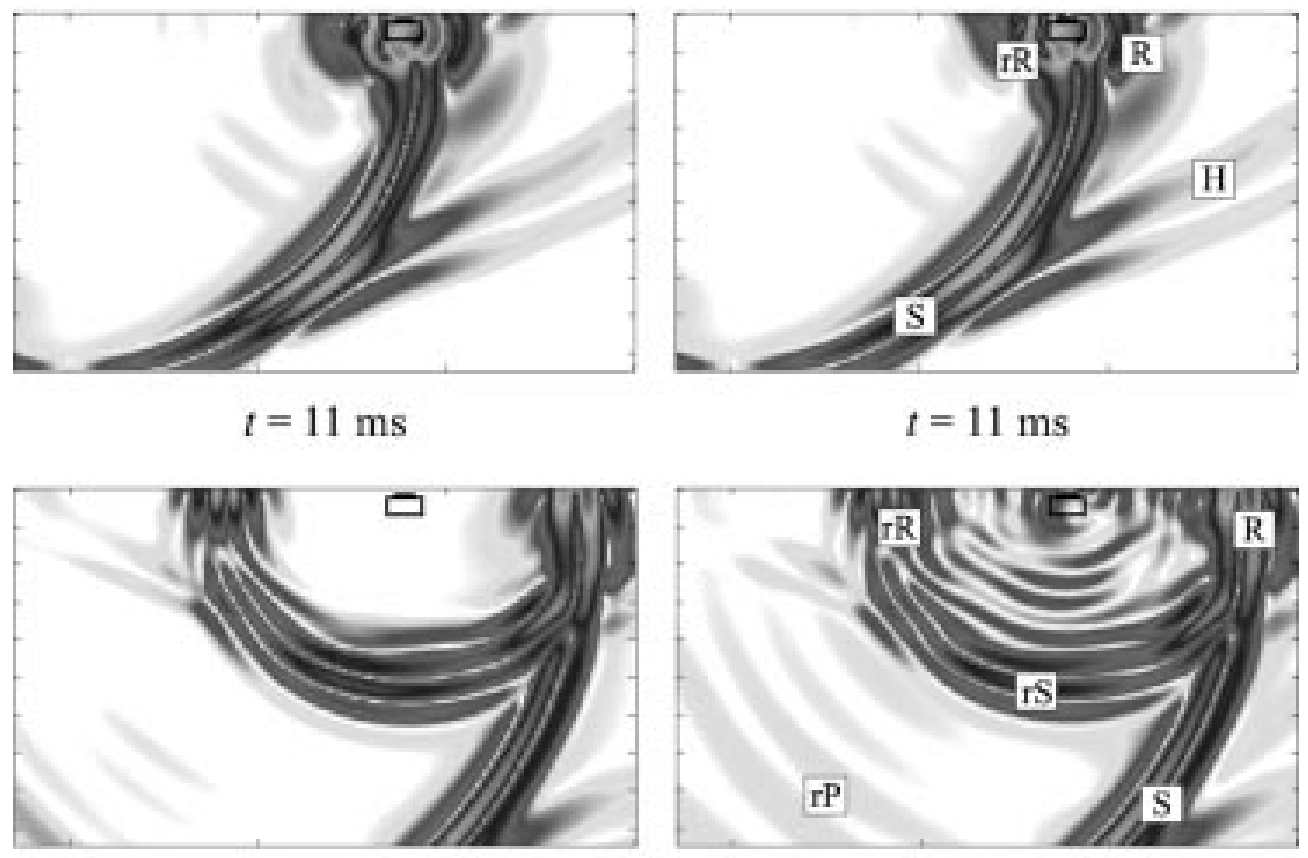

$$
t=16 \mathrm{~ms}
$$

(a)

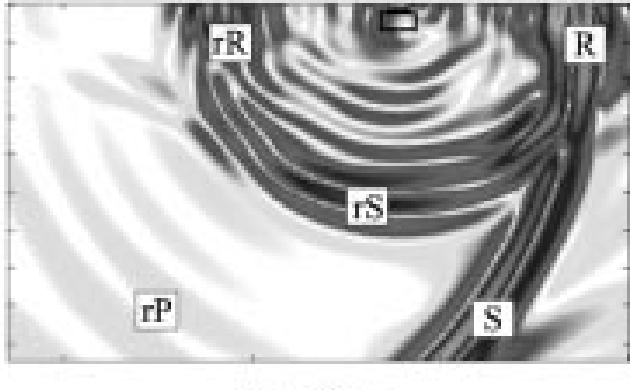

$$
t=16 \mathrm{~ms}
$$

(b)

Fig. 6. Interaction of elastic waves with a buried antipersonnel mine and pseudo-color plots of the magnitude of the particle velocity on a cross section through the ground. (a) Mine with air-filled chamber. (b) Mine without air-filled chamber. R: Rayleigh wave; S: Shear wave; P: pressure wave; H: Head wave; rR: reflected Rayleigh wave; rS: reflected Shear wave; rP: reflected pressure wave. The arrow in the upper two plots denotes the source location.

onto the mine. The pressure wave is reflected and transmitted by the mine. It converts into a reflected pressure wave, a reflected surface wave, a transmitted pressure wave $(\mathrm{P})$, and a transmitted surface wave $(\mathrm{R})$. These waves are weak, due to the limited energy content of the pressure wave near the surface. The pressure wave is seen to travel faster over the mine than in the sand. 


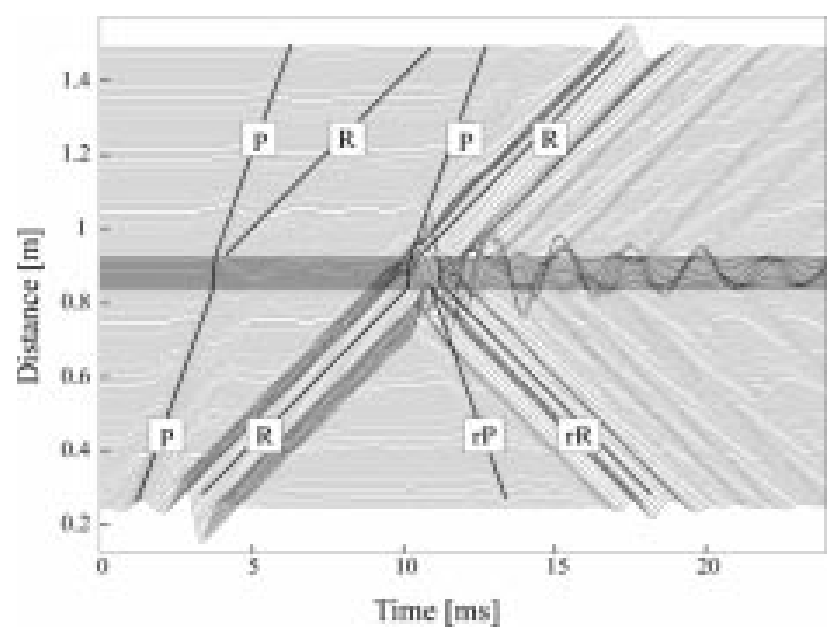

(a)

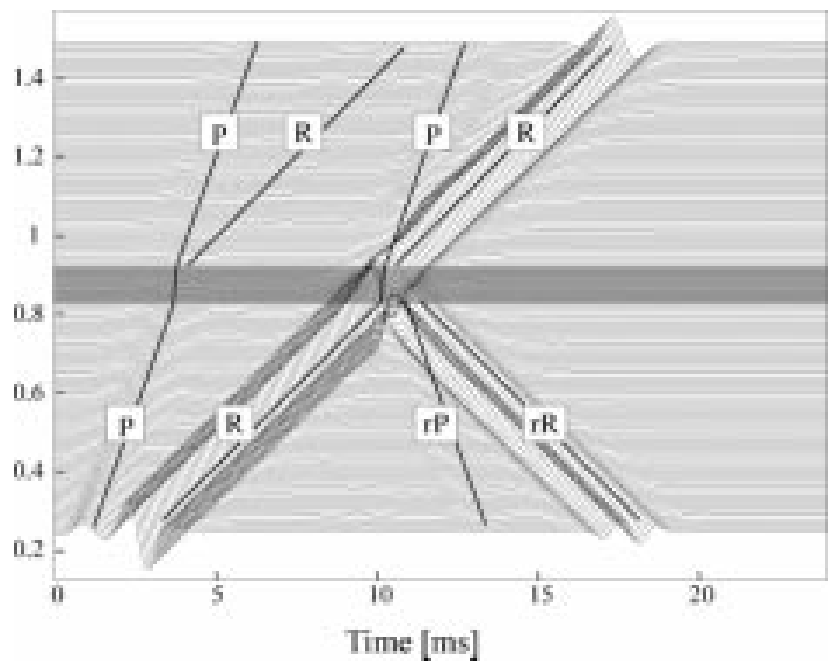

(b)

Fig. 7. Interaction of elastic waves with a buried antipersonnel mine and waterfall graph of the vertical particle velocity at the surface. (a) Mine with air-filled chamber and (b) mine without air-filled chamber. R: Rayleigh wave; P: pressure wave; rR: reflected Rayleigh wave; rP: reflected pressure wave. The gray box indicates the mine location.

This is due to the higher wave speed in the mine. The incident surface wave gives rise to a reflected pressure wave $(\mathrm{rP})$, a reflected surface wave $(\mathrm{rR})$, a transmitted pressure wave $(\mathrm{P})$, and a transmitted surface wave (R). For the mine with the air-filled chamber [Fig. 7(a)], a strong resonance can be seen at the mine location. The resonance remains at the mine even after the incident surface wave has passed by, and causes the mine to radiate. This resonance is due to energy being trapped between the mine and the surface. The nature of this resonance will be explored further in Section III-B. For the mine without the airfilled chamber [Fig. 7(b)], no resonance occurs.

\section{A. Comparison to Experimental Results}

The results obtained with the numerical model are in fairly good agreement with experimental results [2]. In the experimental setup, an inert TS-50 antipersonnel mine (see Fig. 5) is buried in sand. A differentiated Gaussian pulse with a center frequency of $450 \mathrm{~Hz}$ is launched by an electrodynamic transducer placed on the surface of the ground, $85 \mathrm{~cm}$ away from

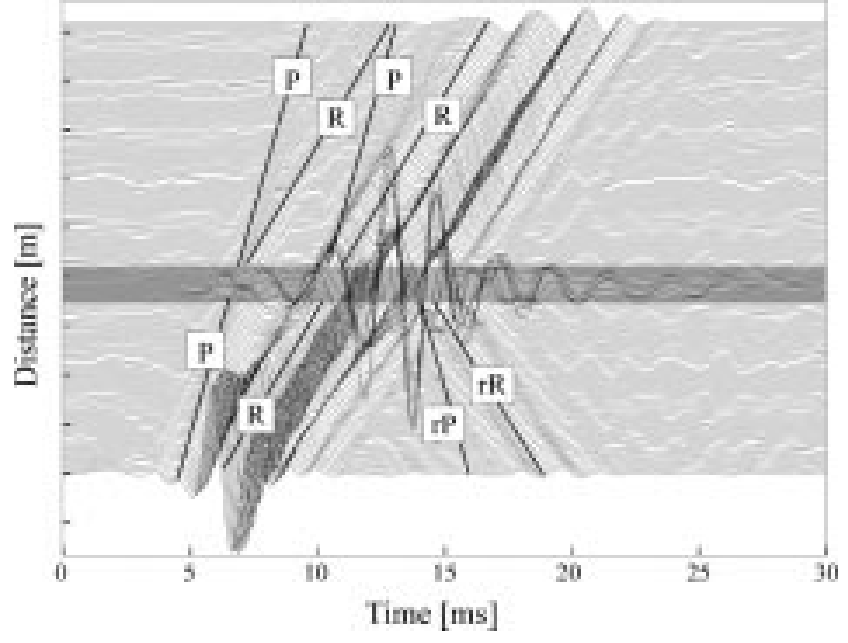

Fig. 8. Interaction of elastic waves with a buried antipersonnel mine (experimental results) and waterfall graph of the vertical particle displacement at the surface. R: Rayleigh wave; P: pressure wave; rR: reflected Rayleigh wave; rP: reflected pressure wave. The gray box indicates the mine location.

the mine. Above the surface, a radar that detects the vibrations of the surface is mounted. Fig. 8 shows a waterfall graph of the vertical particle displacement ${ }^{2}$ on the surface as obtained in measurements with an inert TS-50 antipersonnel mine. The mine is buried $1.3 \mathrm{~cm}$ beneath the surface. The shear wave speed and the pressure wave speed were measured to be $87 \mathrm{~m} / \mathrm{s}$ and $250 \mathrm{~m} / \mathrm{s}$, respectively. A pressure wave $(\mathrm{P})$ and a Rayleigh wave $(\mathrm{R})$ are seen to propagate and to interact with the buried mine. A resonance very similar to the one obtained in the numerical simulation of the mine with the air-filled chamber is observed. In the experimental model, the resonance appears stronger than in the numerical model, whereas in the numerical model, the reflections from the mine are much stronger. These differences are mainly due to the numerical model being 2-D, whereas the experimental model is 3-D. Furthermore, the model for the mine used in the numerical simulation is very simple and approximates the complex structure of a real land mine only very coarsely.

In the experiment, the Rayleigh surface wave is seen to be dispersed as it travels along the surface. This is believed to be due to the shear wave speed varying with depth. Due to increased cohesion of the sand, the sand becomes stiffer if subjected to increased pressure and thus, the shear wave speed increases with depth [13]. Empirically, the depth dependence of the sand has been estimated to be

$$
c_{s}=\left[70+88 \cdot z^{0.3}\right] \mathrm{m} / \mathrm{s}
$$

where $z$ is the depth in meters. Using this depth dependence within the numerical model, the Rayleigh wave disperses in a very similar manner as observed experimentally (Fig. 9).

\section{B. Resonance}

Both in the experiment and in the numerical simulation, a strong resonance is seen at the mine location. Though the nu-

\footnotetext{
${ }^{2}$ Note that experimentally, the particle displacement is measured, whereas the particle velocity is determined in the numerical model. Since the particle velocity is just the time derivative of the particle displacement, both wave fields bear the same characteristic behavior.
} 


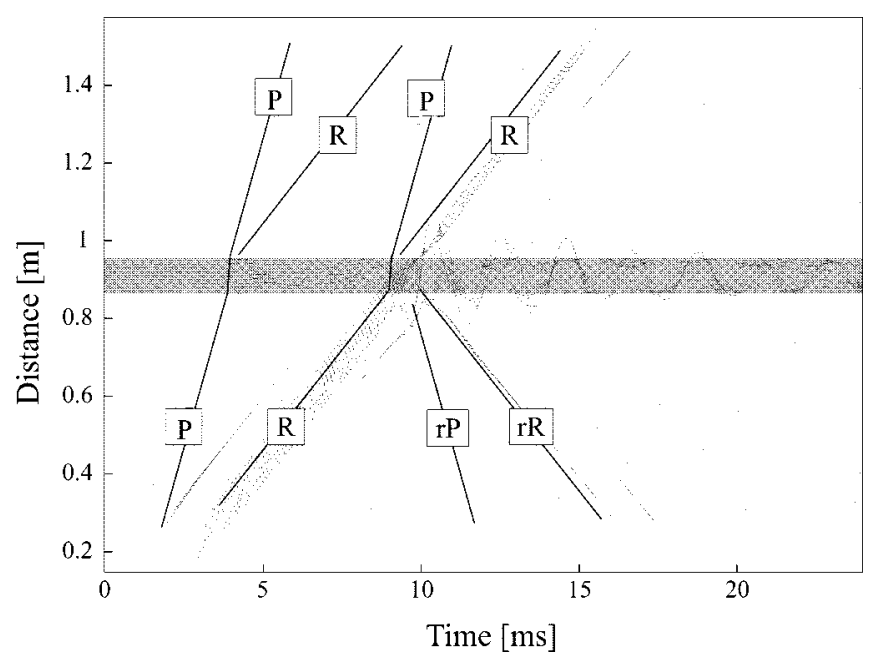

Fig. 9. Interaction of elastic waves with a buried antipersonnel mine (numerical simulation for a mine with air filled chamber) and waterfall graph of the vertical particle velocity at the surface (mine with air-filled chamber) The shear wave speed within the ground varies with depth and causes the waves to disperse. R: Rayleigh wave; P: pressure wave; rR: reflected Rayleigh wave; $r P$ : reflected pressure wave. The gray box indicates the mine location

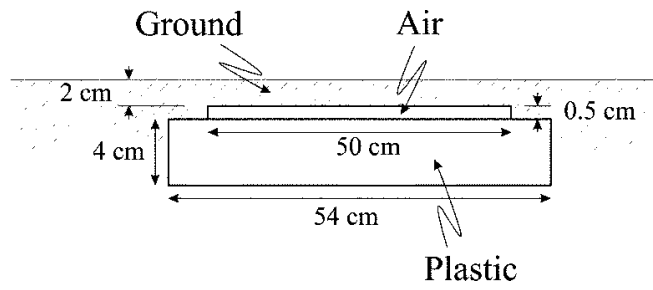

Fig. 10. Model to determine the nature of the resonance (elongated mine).

merical model for the mine is very simple, the shape of the resonance in the experiment strongly resembles the resonance obtained in the simulation. In order to explore the origin of the resonance and its nature, the mine is elongated within the numerical model, as shown in Fig. 10.

The resulting waves are shown in Fig. 11. The vertical particle velocity on the surface is depicted in a waterfall graph. As before, the waves can be easily distinguished by comparing their wave speeds. Pressure waves $(\mathrm{P})$, Rayleigh waves $(\mathrm{R})$, and head waves $(\mathrm{H})$ are seen to propagate. They hit the mine and are partially reflected and partially transmitted. However, above the mine, a complex pattern of propagating waves arises. This can be explained as follows. First, the pressure wave hits the mine. The pressure wave couples its energy mostly into three different wave portions: reflected waves, waves that are transmitted through the plastic body underneath the air chamber, and waves that propagate within the thin layer between the surface and the air chamber. Because the plastic body lies underneath the air chamber, the wave portion transmitted through the plastic is not visible on the surface. This is evident in Fig. 11, where a transmitted pressure wave arises beyond the mine, apparently without being caused by any wave visible on the surface above the air chamber (the path the wave takes through the plastic is indicated by Pm). The Rayleigh wave carries much more energy than the pressure wave. When the Rayleigh wave hits the

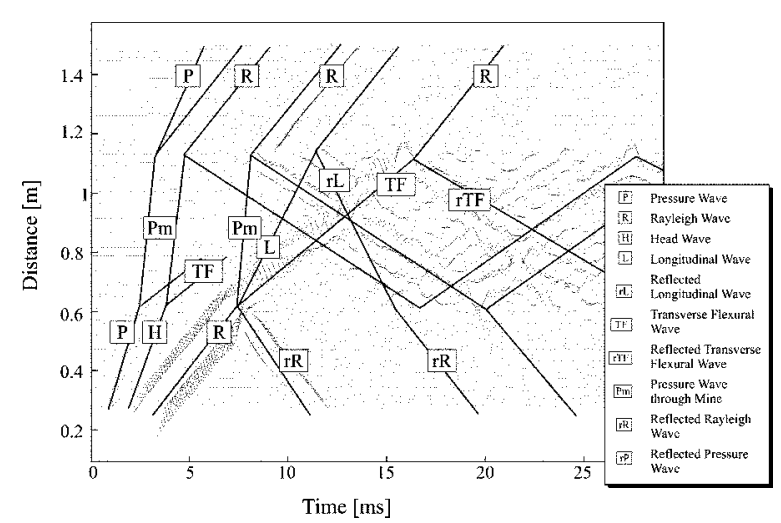

Fig. 11. Waves in the thin layer between the surface and the air chamber and waterfall graph of the vertical particle velocity.

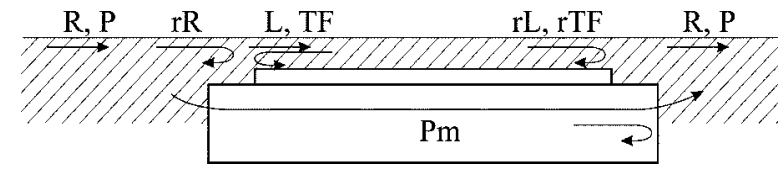

Fig. 12. Waves in the thin layer between the surface and the air chamber. The waves are almost totally reflected at the edge of the air chamber and are thus trapped within the thin layer above the air chamber.

mine, the same kinds of waves arise as for the incident pressure wave: reflected waves (pressure and Rayleigh waves, marked by $\mathrm{rP}$ and $\mathrm{rR}$ ), waves transmitted through the plastic body (pM), and waves propagating within the thin layer between surface and air chamber. The waves through the plastic body behave as described for the pressure wave. However, the Rayleigh wave couples a significant amount of energy into the thin layer above the air chamber. Two different kinds of waves arise within the thin layer: a longitudinal wave and a transverse flexural wave. The longitudinal wave is the faster one of the two. It propagates within the thin layer between surface and air chamber (L), is reflected at the edge of the air chamber (rL), travels back within the layer and is reflected again. The transverse flexural wave also travels within the layer between surface and air chamber (TF), is reflected (rTF), travels back, and is again reflected. Note that the flexural waves are almost totally reflected at the edges of the air chamber, and the energy remains within the thin layer between surface and air chamber. Fig. 12 gives a schematic drawing of the principle behavior of the arising waves.

Using this model, the resonant behavior of the mine in the numerical model can be explained. When the waves hit the mine, they are partially reflected and partially transmitted. However, a large portion of the energy couples over into longitudinal and transverse (flexural) waves propagating within the thin layer between the surface and the mine's air chamber. Due to the short length of the mine, these waves form standing waves.

From the slope of the traveling waves in Fig. 11, the wave speeds of the longitudinal wave and the transverse flexural wave are determined to be $c_{L} \approx 160 \mathrm{~m} / \mathrm{s}$ and $c_{T F} \approx 50 \mathrm{~m} / \mathrm{s}$, respectively. A simple theory for waves propagating in a thin plate predicts the wave speeds of the longitudinal wave to be [10]

$$
c_{L}=\left(\frac{E}{\rho\left(1-\nu^{2}\right)}\right)^{1 / 2}
$$


and the wave speed of the transverse flexural wave to be

$$
c_{T F}=\sqrt{h \omega\left(\frac{E}{12 \rho\left(1-\nu^{2}\right)}\right)^{1 / 2}}
$$

where $E$ and $\nu$ are Young's modulus and Poisson's ratio, respectively, $\rho$ is the material density, and $h$ is the thickness of the thin plate. For the longitudinal wave, this yields a wave velocity of $c_{L} \approx 163 \mathrm{~m} / \mathrm{s}$. For a transverse flexural wave with a center frequency $f_{c}=450 \mathrm{~Hz}$ and for a plate thickness of $h=$ $2 \mathrm{~cm}$, a wave speed of $c_{T F} \approx 52 \mathrm{~m} / \mathrm{s}$ is predicted. This is in good agreement with the wave speeds obtained from the numerical simulation. Note that the wave speed of the transverse flexural wave is dependent on frequency, while the speed of the longitudinal wave is not. Thus, the transverse wave is strongly dispersive. The dispersion of the transverse flexural wave can be clearly seen in Fig. 11.

Note that the analysis of the resonant behavior of an actual mine is much more complex, and the argument outlined here gives only one possible cause for the occurring resonance. An actual mine is 3-D, has a flexible case that can support both flexural and longitudinal waves, and contains springs that can also give rise to resonances. The authors believe that the resonances observed in the experiments are due to flexural waves being trapped in both the case of the mine and the layer of soil above the mine. Experimentally, resonances are observed in mines flush with the surface of the ground. The authors are working to improve the model so that it more accurately predicts the mine-wave interactions.

\section{CONCLUSIONS}

A finite-difference model has been developed and implemented in 2-D. An analytical solution was used to validate the finite-difference model. The finite-difference model was used to investigate the interaction of elastic waves with a buried antipersonnel mine. The TS-50 antipersonnel mine was approximated by two different models: one with an air-filled chamber and one without an air-filled chamber. Even though the numerical model was very simple, the results from the numerical model were found to be in good qualitative agreement with experimental results. Both in the experiment and in the numerical model containing the air-filled chamber, a strong resonanance at the mine location occured, whereas the model without the air-filled chamber did not show resonant oscillations. The nature of the resonance was investigated by slightly modifying the numerical model. If the air-chamber is elongated, flexural waves are seen to be excited and to propagate within the thin layer between the surface and air chamber. These waves are almost totally reflected at the edges of the air chamber and are thus trapped within the thin layer. In these experiments, the Rayleigh wave was seen to disperse as it travels along the surface. By assuming a depth-dependent shear wave speed, this effect can be reproduced within the numerical model.

\section{REFERENCES}

[1] W. R. Scott, Jr., C. T. Schröder, and J. S. Martin, "An hybrid acousto/electromagnetic technique for locating land mines," in Proc. Int. Geoscience and Remote Sensing Symp., Seattle, WA, 1998, pp. 216-218.

[2] W. R. Scott, Jr. and J. S. Martin, "Experimental investigation of the acousto-electromagnetic sensor for locating land mines," Proc. SPIE, vol. 3710, pp. 209-214, Apr. 1999.

[3] C. T. Schröder, "A Finite-Difference Model for Elastic Waves in the Ground," Diploma thesis, Univ. Braunschweig, Braunschweig, Germany, 1999.

[4] J. Virieux, "SH-wave propagation in heterogenous media: Velocity-stress finite-difference method," Geophys., vol. 49, pp. 1933-1957, Nov. 1984.

[5] J. Virieux, "P-SV wave propagation in heterogenous media: Velocitystress finite-difference method," Geophys., vol. 51, pp. 889-901, Apr. 1986.

[6] J.-P. Berenger, "A perfectly matched layer for the absorption of electromagnetic waves," J. Comput. Phys., vol. 114, pp. 185-200, 1994.

[7] W. C. Chew and Q. H. Liu, "Perfectly matched layer for elastodynamics; a new absorbing boundary condition," J. Comput. Acoust., vol. 4, pp. 341-359, 1996.

[8] S. D. Gedney, "An anisotropic perfectly matched layer-absorbing medium for the truncation of FDTD-Lattices," IEEE Trans. Antennas Propagat., vol. 44, pp. 1630-1639, Dec. 1996.

[9] G. F. Miller and H. Pursey, "The field and radiation impedance of mechanical radiators on the free surface of a semi-infinite isotropic solid," Proc. R. Soc. London, vol. A 223, pp. 521-541, 1954.

[10] K. F. Graff, Wave Motion in Elastic Solids: Dover Publications, 1975.

[11] A. Tavlove, Computational Electromagnetics: The Finite-Difference Time-Domain Method. Norwood, MA: Artech House, 1995.

[12] M. Roth, R. Spitzer, and F. Nitsche, "Seismic survey across an environment with very high poisson's ratio," in Proc. 12th Annu. Int. Meeting., Symp. Application of Geophysics to Engineering and Environmental Problems, 1999, pp. 49-55.

[13] B. M. Das, Principles of Soil Dynamics. Boston, MA: PWS-Kent, 1993.

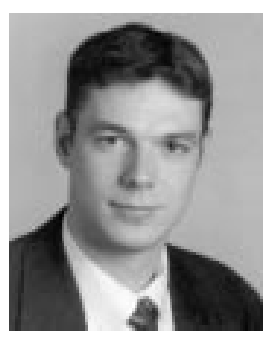

Christoph T. Schröeder was born in Bonn, Germany, in 1973. He received the M.S. degree in electrical engineering from the Georgia Institute of Technology, Atlanta, in 1997, and the Diplom Elekt. from the Technical University of Braunschweig, Braunschweig, Germany, in 1999. He is currently pursuing the $\mathrm{Ph} . \mathrm{D}$. degree in electrical engineering at the Georgia Institute of Technology.

His research interests include elastic wave propagation in solids, remote sensing, surface-acoustic wave devices, electromagnetics, and the finite-difference time-domain technique.

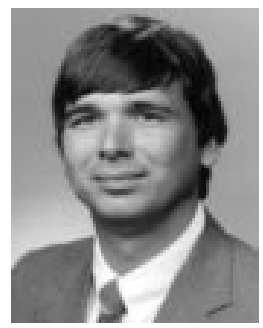

Waymond R. Scott, Jr. received the B.S., M.S., and $\mathrm{Ph} . \mathrm{D}$. degrees, all in electrical engineering, from the Georgia Institute of Technology, Atlanta, in 1980, 1982, and 1985, respectively.

He joined the Faculty of the School of Electrical Engineering, Georgia Institute of Technology, in 1986, where he is currently an Associate Professor of Electrical Engineering. His current research interests include the development of new techniques for measuring the electric and magnetic properties of materials, the development of materials with adjustable constitutive parameters, transient electromagnetic fields, ground penetrating radar, subsurface sensing, and numerical techniques such as the finite element and the finite-difference time-domain techniques. 